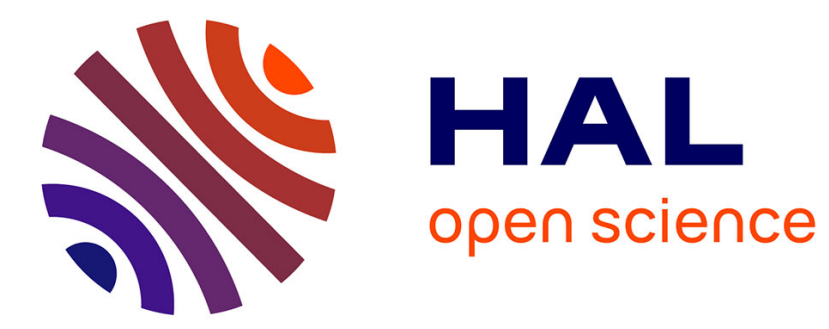

\title{
Edge enhancement by local deconvolution
}

Olivier Laligant, Frédéric Truchetet, Alain Dupasquier

\section{To cite this version:}

Olivier Laligant, Frédéric Truchetet, Alain Dupasquier. Edge enhancement by local deconvolution. Pattern Recognition, 2005, 38, pp.661-672. 10.1016/j.patcog.2004.10.006 . hal-00580932

\section{HAL Id: hal-00580932 \\ https://u-bourgogne.hal.science/hal-00580932}

Submitted on 30 Mar 2011

HAL is a multi-disciplinary open access archive for the deposit and dissemination of scientific research documents, whether they are published or not. The documents may come from teaching and research institutions in France or abroad, or from public or private research centers.
L'archive ouverte pluridisciplinaire HAL, est destinée au dépôt et à la diffusion de documents scientifiques de niveau recherche, publiés ou non, émanant des établissements d'enseignement et de recherche français ou étrangers, des laboratoires publics ou privés. 


\title{
Edge enhancement by local deconvolution
}

\author{
O. Laligant*, F. Truchetet, A. Dupasquier \\ Le2i Lab. - UMR CNRS 5158 \\ IUT \\ 12, rue de la Fonderie \\ 71200 Le Creusot \\ FRANCE
}

\begin{abstract}
In this paper a new approach for blurred image restoration is presented. Our algorithm is based on human vision which zooms back and forth in the image in order to identify global structures or details. Deconvolution parameters are estimated by an edge detection and correspond to the ones of a chosen edge detection model. The segmentation is obtained by merging multiscale information provided by multiscale edge detection. The edge detection is achieved by using a derivative approach following a generalization of Canny-Deriche filtering. This multiscale analysis performs an efficient edge detection in noisy blurred images. The merging leads to the best local representation of edge information across scales. The algorithm deals with a mixed (coarse-to-fine/fine-to-coarse) approach and searches for candidate edge points through the scales. Edge characteristics are estimated by the merging algorithm for the chosen model. Scale, direction and amplitude informations allow a local deconvolution of the original image. The noise problem is not considered in this work since it does not disturb the process. Results show that this method allows non-uniformly blurred image restoration. An implementation of the whole algorithm in a intelligent camera (DSP) has been performed.
\end{abstract}




\section{Introduction}

Shooting a real world image with a camera through an optical device gives a 2D image where at least some parts are affected by a blur. This optical blurring can generally be correctly modeled by the effect of a Gaussian filtering on the image. Furthermore, the blur of real edges can have other sources than optical ones: movement of the item in the scene, coarsiness of resolution in the imaging process, effect of diffuse lighting, etc. Restoration of blurred images is one of the main topics in image processing and many methods have been described in literature. Generally these methods are based on a filtering process, the major difficulty in image restoration being the lack of knowledge about the degradation process [1]. In some methods the degradation is assumed to be well known and the restoration problem is reduced to the inversion of the degradation process $[2,3]$. In some other approaches the degradation is supposed to be uniform and the method tends to estimate both the original image and the blur from the degraded image characteristics $[4,5,6]$. Our present work lies at the frontier between these two approaches. We assume that the blur degradation process is according to a new parametric model. This model is an edge profile characterized by three parameters: scale, amplitude and orientation. The principle of the proposed restoration method (IBEP: identifying blur edge profile) consists in locally identifying the edge profile parameters

Email address: email: o.laligant@iutlecreusot.u-bourgogne.fr (O. Laligant*,). 
and then enhancing the edge by a local deconvolution. Identification of various profiles is achieved by a multiscale edge detection assisted by a fusion process to eliminate the problems of over detection and mutual influence (delocalization). This stage produces an image of edge points associated to profile parameters. These data are then used for local and stable deconvolution. The figure 2 presents the overview of the method. Note that the method operates independantly from the noise and does not affect the signal to noise ratio. This non iterative method has been developed and implemented to achieve a real-time autonomous system.

In the first section, we present the blur profile model as well as the operator used for edge detection. The second section is dedicated to the scheme of multiscale edge detection including the phase of fusion. This stage deals with the mutual influence problem and produces information used to estimate the local model. The enhancement method illustrated with practical results and a comparison with the well known and classical Wiener filtering approach are presented in the last section.

\section{Edge detection}

\subsection{Edge model}

The profile of an edge affected by blurring is modelled by a low-pass exponential function. The normalized edge profile model (figure 1) is defined by equation (1): 


$$
C_{s_{c}}(u)= \begin{cases}1-\frac{e^{-s_{c} u}}{2} & u \geq 0 \\ \frac{e^{s_{c} u}}{2} & u<0\end{cases}
$$

This function can be seen as a first-order approximation of the majority of edges encountered in real image processing and it corresponds to the filtering of an ideal step edge by a low-pass filter with impulse response: $h(u)=\frac{s_{c}}{2} e^{-s_{c}|u|}$. A somewhat more realistic model should be a step function filtered by a gaussian filter (erf function) but its form is far less suitable for mathematic treatment than the proposed approximation. The parameter $s_{c}$ represents the scale of the blur. The smaller the parameter value is, the greater the blur is. The amplitude of the edge will be denoted by $A_{c}$ and its orientation with respect to image lines direction by $\theta_{c}\left(u\right.$ is the abscissa in the direction $\left.\theta_{c}\right)$. Hence, an edge profile $E P_{c}$ in the image is characterized by three parameters and will be denoted as:

$$
E P_{c}=\left(s_{c}, A_{c}, \theta_{c}\right)
$$

The estimation of these three parameters is achieved on the basis of a multiscale edge detection method.

\subsection{Edge detector}

The restoration process is always more or less equivalent to high pass filtering. It is well known that such an operation is generally unstable and very sensitive to high frequency corrupting signal (noise). In our design, in order to avoid this drawback, the restoration process is applied only where a true edge is present. Therefore these edges have to be detected and localized, even if the 
image is noisy. This task is realized by optimal edge detection such as the one proposed by Canny and Deriche or Shen and Castan. These filters are designed to give a optimum response with respect to constraints about signal over noise ratio, edge localization, multiple responses suppression. We have shown in previous works that these filters can be seen as special cases of a familly of filters where response is optimal for the edge model proposed in the previous section. These filters are parametrized by a scale factor and they lead to a multiscale gradient estimator. The prototype impulse response of the generalized filter family [7] is defined as following:

$$
f_{n, j}(x)=-\operatorname{sign}(x)^{n+1} \frac{s_{j}^{n+1}}{n !} x^{n} e^{-s_{j}|x|}
$$

where $n$ is the index of the filter and $j$ the scale level. We choose the scale factor $s_{j}$ as:

$$
s_{j}=\frac{s}{a^{j}}
$$

with $s, a>0$ and $j \in \mathbf{Z}$.

Shen \& Castan[8] and Canny-Deriche [9, 10] filters correspond respectively to $n=0$ and $n=1$. Each filter is normalized with respect to unit step response:

$$
\left|\int_{-\infty}^{0} f_{n, j}(\tau) d \tau\right|=\left|\int_{0}^{+\infty} f_{n, j}(\tau) d \tau\right|=1
$$

\subsection{Responses and performances of the detectors}

The filter is to be applied on the image in order to detect, localize and identify (determine the parameters) the edges. 
It is therefore necessary to analyze its response and performances with respect to the blur scale $s_{c}$.

\subsubsection{Response}

The response maximum $y(0)$ of these detectors to the edge model is (see details in appendix A):

$$
\begin{aligned}
y_{n, j}(0) & =f_{n, j} * C_{s_{c}}(0) \\
& =1-\frac{s_{j}^{n+1}}{\left(s_{j}+s_{c}\right)^{n+1}}
\end{aligned}
$$

This result will be used to design the enhancement process (section 4). This maximum decreases as the scale factor $s_{j}$ increases for $s_{c}$ fixed. It increases as the blur parameter $s_{c}$ increases for $s_{j}$ fixed.

\subsubsection{Edge localization}

The definition given by Canny [11] of a criterion for localization of an edge $C$ in presence of additive white Gaussian noise of variance unity and detected by an operator $f$ is as following:

$$
L(f)=\frac{\left|\int_{-\infty}^{+\infty} C_{s_{c}}^{\prime}(-\tau) f^{\prime}(\tau) d \tau\right|}{\left(\int_{-\infty}^{+\infty} f^{\prime 2}(\tau) d \tau\right)^{\frac{1}{2}}}
$$

therefore (see details in appendix B), for $n=1,2,3, \ldots$

$$
L\left(f_{n, j}\right)_{n \in\{1,2, \ldots\}, j \in \mathbf{Z}}=\frac{s_{c}^{2}}{\left(s_{j}+s_{c}\right)^{n+1}} \sqrt{\frac{2 n-1}{s_{j}(2 n) !}}\left(2 s_{j}\right)^{n} n !
$$


Taking into account the 0 -order discontinuity at $x=0$ for $n=0$

$$
L\left(f_{0, j}\right)_{j \in \mathbf{Z}}=\frac{s_{c}}{s_{j}+s_{c}} \sqrt{s_{j}}
$$

For each detector, the localization criterion presents a maximum according to the detection scale $s_{j}$. We define the optimal scale $S_{n}$

$$
\left\{S_{n}=\arg \left[\max _{s_{j}}\left(L_{n}\left(s_{j}\right)\right)\right]\right\}_{n \in\{1,2, \ldots\}}
$$

Value $S_{n}$ of $s_{j}$ corresponding to the maximum in localization depends on the blur scale $s_{c}$ and naturally on the index $n$ of the used detector. On both sides of this extremum, localization criterion tends toward 0 when $s_{j} \rightarrow 0$ and when $s_{j} \rightarrow \infty$. The scale of the detector for this maximum in localization is given for $n \in\{1,2,3, \ldots\}$ by (see appendix D):

$$
\left\{S_{n}=\frac{1}{3}(2 n-1) s_{c}\right\}_{n \in\{1,2, \ldots\}}
$$

and for $n=0$ :

$$
S_{0}=s_{c}
$$

\subsubsection{Signal to noise criterion}

The definition given by Canny for signal to noise ratio in detection of an

edge $C$ in the presence of additive white Gaussian noise of variance unity and filtered by an operator $f$ is as following: 


$$
\Sigma(f)=\frac{\left|\int_{-\infty}^{+\infty} C_{s_{c}}(-\tau) f(\tau) d \tau\right|}{\left(\int_{-\infty}^{+\infty} f^{2}(\tau) d \tau\right)^{\frac{1}{2}}}
$$

therefore (see details in appendix C)

$$
\Sigma\left(f_{n, j}\right)_{n \in\{0,1,2, \ldots\}, j \in \mathbf{Z}}=\frac{\left(s_{j}+s_{c}\right)^{n+1}-s_{j}^{n+1}}{\left(s_{j}+s_{c}\right)^{n+1}} \frac{2^{n} n !}{\sqrt{s_{j}(2 n) !}}
$$

For an edge scale $s_{c}$, this criterion is maximum for $s_{j}=0$ and decreases when $s_{j}$ increases. The evolution of this criterion is naturally connected to the maximal response presented above.

\subsection{Strategy for restoration}

The principle of restoration method IBEP (identifying blur edge profile) is based on the identification of the model for edge profiles in the image. This identification consists in estimating $E P_{c}$. The purpose of the restoration is to enhance the edges by local deconvolution to obtain a step profile:

$$
E P_{c}^{\infty}=\left(A_{c}, \theta_{c}\right)
$$

A detection on two scales at least is necessary to determine the scale $s_{c}$ and the amplitude $A_{c}$ of $E P_{c}$, orientation being deduced from the gradient direction information. A study of the proposed detectors shows on one hand that the best localization of edge is obtained for a detection scale given by equations 12,11 . On the other hand, the immunity to noise increases as the scale of detection decreases. It is the same for the maximum response. However, for 
too small scales, localization falls very quickly.

As blur is assumed to be variable in the image, we have to choose for detection an interval of scales $s$ close to the range of blur scales $s_{c}$.

From this analysis we can conclude that for detection, localization and characterization of the edges a strategy has to be followed. This strategy in a multiresolution approach consists in choosing a set of detection scales with a geometric regular step (noted $a$ ) compatible with expected blur in the image, then from the detection scales close to the maximum of localization, in determining exactly the scale, the direction and the amplitude of the best local model.

\section{Edge image representation}

\subsection{Multiscale edge detection}

For each point $I(x, y)$ of the image, the gradient vector is obtained by a $2 \mathrm{D}$ separable convolution. Following J. Canny[11], let $h_{n, j}$ be the regularization operator associated with $f_{n, j}: \frac{d h_{n, j}(x)}{d x}=f_{n, j}(x)$. A 2D separable operation is defined as: $h_{n, j}(x, y)=h_{n, j}(x) \cdot h_{n, j}(y)$. Therefore the gradient vector $\overrightarrow{G(x, y)}$ is defined as: 


$$
\begin{aligned}
\overrightarrow{G(x, y)}=\left(\begin{array}{c}
G_{x}(x, y) \\
G_{y}(x, y)
\end{array}\right) & =\left(\begin{array}{c}
\frac{d}{d x}\left(h_{n, j}(x, y) * I(x, y)\right) \\
\frac{d}{d y}\left(h_{n, j}(x, y) * I(x, y)\right)
\end{array}\right)_{n \in\{0,1, \ldots\}, j \in[0,1, \ldots, p-1]} \\
& =\left(\begin{array}{l}
f_{n, j}(x) h_{n, j}(y) * I(x, y) \\
h_{n, j}(x) f_{n, j}(y) * I(x, y)
\end{array}\right)_{n \in\{0,1, \ldots\}, j \in[0,1, \ldots, p-1]}
\end{aligned}
$$

It is easy to compute the edge orientation: $\theta=\operatorname{Arg}[\overrightarrow{G(x, y)}]$. Multiscale analysis is achieved by a multiscale set of $p$ operators $\left\{f_{n, j}\right\}_{j \in[0,1, \ldots, p-1]}$ of scales $s_{j}=\frac{s}{a^{j}}$ with $j \in[0,1, \ldots, p-1], s$ being initial scale and $a>0$. In this way, a set of $p$ gradient images is obtained: $\left\{\vec{G}_{j}\right\}_{j \in[0,1, \ldots, p-1]}$. Fine scales and coarse scales correspond respectively to small and large values of $s_{j}$.

Candidate edge points $\left\{L_{j}(x, y)\right\}_{j \in[0,1, \ldots, p-1]}$ in the gradient images are localized by extraction of local maxima. After detection and localization, the next step (see 2) consists in estimating $s_{c}$ and $A_{c}$ in the original image. However, one observes variations of localization and amplitude due not only to noise but also to influence of neighbor edges. This phenomenon has not been really analyzed in the literature dedicated to edge detection. In the next section, we propose a simple and pratical study leading to some rules allowing these perturbations to be avoided or taken into account in the restoration process.

\subsection{Influence of a neighbor edge}

It is the distortion induced by a neighbor edge on the detection of the current edge that is under study. To simplify the discussion of this influence we con- 
sider only infinite scale edge profiles. However, the observations will remain valid whatever may be the scales. Two kinds of influence can be distinguished depending on the respective configuration of the two edges. Opposite edges lead to a substractive influence while two edges in a staircase-like configuration give an additive influence.

- Firstly we have a configuration of two parallel opposite edges (figure 3), current profile $E P_{c}^{\infty}=\left(\infty, A_{c}>0, \theta_{c}\right)$ and neighbor profile $E P_{v}^{\infty}=\left(\infty, A_{v}<\right.$ $\left.0, \theta_{v}=\theta_{c}\right) \cdot h_{n}$ is the step response to the detector $f_{n}$. The response to $E P_{c}^{\infty}$ is the superposition of two responses (to $E P_{c}^{\infty}$ alone and $E P_{v}^{\infty}$ alone):

$$
D_{n, j}(x)=A_{c} h_{n, j}(x)+A_{v} h_{n, j}(d-x)
$$

$d$ being the distance between the two edges and

$$
h_{n, j}(x)=e^{-s_{j}|x|} \sum_{i=0}^{n} \frac{s_{j}^{n-i}}{(n-i) !}|x|^{n-i}
$$

An edge is assumed to be detected where response is maximum. Therefore an edge of amplitude $A_{c}$ is detected at location $x_{c}$ close to 0 if there exists a zero-crossing in the range $] x_{c} ; x_{v}$ [ where $x_{v}$ is the location of the detection of a neighbor profile; $x_{c}$ is negative or null $\left(x_{c} \leqslant 0\right)$. Absolute value of $x_{c}$ increases and the amplitude of the response maximum decreases as the influence of the neighbor edge increases.

Proposition 1 Subtractive influence is the influence of a neighborhood of gradient sign opposite to the current point one. It tends to decrease the detection amplitude as the detector scale decreases and it displaces the highest response maximum toward the fine scales.

- Secondly, let us consider the other configuration where edges profiles form a stair (figure 4): $E P_{c}^{\infty}=\left(\infty, A_{c}>0, \theta_{c}\right)$ and $E P_{v}^{\infty}=\left(\infty, A_{v}>0, \theta_{v}=\theta_{c}\right)$. 
In this case, the profile $E P_{c}^{\infty}$ of amplitude $A_{c}$ will be detected if there exists a minimum between two maximum responses to two profiles $E P_{c}^{\infty}$ and $E P_{v}^{\infty}$ (so that they can be separated).

The influence of the neighbor edge tends to displace the response to current edge: in that case $x_{c}$ is positive. The amplitude of the response maximum increases as the influence of the neighbor edge increases (detection scale decreases). This remark leads to a second proposition:

Proposition 2 Additive or amplification influence is the influence due to a neighborhood whose gradient sign is the same as the current point one. It tends to increase the detection amplitude as the detector scale decreases and it displaces the highest response maximum toward the coarse scales.

Remark 1 Due to its infinite derivative at 0, Shen 8 Castan filter does not delocalize the responses whatever the influence. Nevertheless, the amplitude increases or decreases following the above propositions.

\subsection{Merging}

Merging, as considered in IBEP (see figure 2) is defined as the operation consisting in the choosing of the scale providing the best local edge representation (at a given pixel) among all available scales [12].

We have seen, in a previous section, how in a first step, a set of gradient $\left\{\vec{G}_{j}\right\}_{j \in[0, \ldots, p-1]}$ and local maximum $\left\{L_{j}\right\}_{j \in[0, \ldots, p-1]}$ images are obtained from the multiscale analysis method. In the next step one has to localize and to characterize local edge profiles $\left(E P_{c}\right)$ by merging these data.

Gradient orientations are coded into eight sectors of 45 degrees according to 
the Freeman code (see figure 8): $\left\{\theta_{0}=\left\{\left[-22.5^{\circ}, 22.5^{\circ}[\}, \theta_{1}=\left\{\left[22.5^{\circ}, 67.5^{\circ}[\}, \theta_{2}=\right.\right.\right.\right.\right.$ $\left\{\left[67.5^{\circ}, 112.5^{\circ}[\}, \ldots \theta_{7}=\left\{\left[292.5^{\circ}, 337.5^{\circ}[\}\right\}\right.\right.\right.$.

According to the discussion on the influence of neighbor edges, it is obvious that subtractive or additive influence will delocalize the current detected edge. This delocalization has to be taken into account. But the initial scale $a$ is assumed to be small enough to avoid any delocalization on the first scale.

The merging algorithm joins the coarse-to-fine approach and the fine-to-coarse one. Coarse-to-fine approach allows to follow the evolution of the tight edge profiles across the scales. The best localization of these edges is performed by this approach. Conversely, fine-to-coarse approach leads to the best detection for wide edge profiles. A good edge characterization of the various profiles occuring in a real world image needs the cooperation of these two approaches.

Local maxima of edge gradient are searched in a neighborhood cone through the scales (figures 6,7). During this search, local maximums under influence of the neighborhood are picked out and eliminated. The greatest amplitudes give the best localization (see figure 5).

The merging algorithm is summarized in the following lines.

- for each current point of the original image, from multiscale gradient information:

- search for the closest maximums in the coarse-to-fine approach. Ordered maximums lead to a first list of candidates points.

- Search for the closest maximums in the fine-to-coarse approach. Ordered maximums lead to a second list of candidates points.

- Discard points under positive influence (see below), points under soustrac- 
tive influence eliminate themselves.

- Through the two ordered lists:

a point is valid if each list represents a maximum at the same scale, same direction and same localization.

A valid point produces an edge if this localization is exactly the position of the current point.

Elimination of points under positive influence is carried out as follows: maximum are sorted in decreasing scales (fine-to-coarse). Two consecutive maximums which do not present the same localization are eliminated.

Following the merging stage, a segmentation method is needed to obtain a binary edge points image. Classic methods like a simple thresholding or the coïncidence assumption of Marr and Hildreth [13] [12] can be used. This last one selects points according to the persistence of the detection across the scales and consequently it is well adapted to our merging method.

Finally, we obtain a list $P_{c}$ of ordered points at the current position $\left(x_{c}, y_{c}\right)$ : $\left.P_{c}=\left\{\left(G x_{j_{A}}, G y_{j_{A}}, s_{j_{A}}\right),\left(G x_{j_{B}}, G y_{j_{B}}, s_{j_{B}}\right), \ldots\right\}\right)$. These points are the best edge information available and represent gradient information.

\section{Restoration}

The IBEP restoration principle is based on a local evaluation in image of scale and amplitude equivalent to the model. The deconvolution based on the model allows to restore the contour. Deconvolution parameters are those of the model $E P_{c}$, they can be deduced from the multiscale edge analysis described in the previous section. 


\subsection{Model parameters estimate}

This part is developped on the basis of the Shen and Castan[8] operator regarding its efficency and simplicity.

Following the notation introduced in the equation 3, the definition of ShenCastan operator is:

$$
f_{0, j}(x)=-\operatorname{sign}(x) s_{j} e^{-s_{j}|x|}
$$

characterized by:

$$
\left\{\begin{array}{c}
y_{0, j}(0)=1-\frac{s_{j}}{s_{j}+s_{c}} \\
L\left(f_{0, j}\right)=\frac{s_{c}}{s_{j}+s_{c}} \sqrt{s_{j}} \\
s_{0}[\max (L)]=s_{c} \\
\Sigma\left(f_{0, j}\right)=\frac{s_{c}}{s_{j}+s_{c}} \frac{1}{\sqrt{s_{j}}}
\end{array}\right.
$$

The above relations are defined in the continuous domain, to improve the precision of the results, discrete versions can be introduced. The normalized filter $\left(\sum_{0}^{+\infty} f_{0, j}[k]=1\right)$ becomes:

$$
f_{0, j}[k]=-N_{s_{j}} \operatorname{sign}[k] e^{-s_{j}|k|}
$$


with $N_{s_{j}}=e^{s_{j}}-1$. The maximum response $y_{0, j}[0]$ to a simple edge profile is:

$$
\begin{aligned}
y_{0, j}[0] & =\sum_{k=-\infty}^{\infty} C_{s_{c}}[l-k] f_{0, j}[k] \\
& =A_{c}\left(1-\frac{N_{s_{j}}}{N_{s_{j}+s_{c}}}\right)
\end{aligned}
$$

with $N_{s_{j}+s_{c}}=e^{s_{j}+s_{c}}-1$.

Four detection scales are processed with a geometrical progression step $a=2$. A minimum of two scales of detection is necessary to estimate the parameters of the current edge profile $E P_{c}$. As presented in section 3.3 edge points are selected by a segmentation method which is the coincidence assumption [13] assisted by a simple threshold. The merging stage produces the list $P_{c}$ with all needful information leading to the profile estimation. $\theta_{c}$ is directly deduced from the gradient information of the first point in the list $P_{c}$. The resolution of a system of equations gives a solution for $A_{c}$ and $s_{c}$.

\subsection{Deconvolution}

The edge profile is a monodimensional object characterized by its amplitude $A_{c}$, its equivalent scale $s_{c}$ and its orientation $\theta_{c} \in\left\{\theta_{0}, \theta_{1}, \ldots, \theta_{7}\right\}$. In the direction $\theta_{c}$, the model profile can be seen as the convolution of a step edge with the following Point Spread Function:

$$
T(u)=\frac{s_{c} e^{-s_{c}|u|}}{2}
$$

where $u$ is the abscissa in direction $\theta_{c}$. Let $I_{0}$ be the original image not affected by blur and $I$ be the degraded image. In direction $\theta_{c}$ : 


$$
I(u)=I_{0} * T(u)
$$

By direct inversion of the blurring operator one obtains a deconvolution function:

$$
T^{-1}(u)=\delta(u)-\frac{1}{s_{c}^{2}} \delta^{\prime \prime}(u)
$$

The enhanced image could be deduced as follows:

$$
\widetilde{I}_{0}(u)=I(u)-\frac{1}{s_{c}^{2}} I^{\prime \prime}(u)
$$

However, as it is well known, the straightforward application of this equation leads to an unstable result whatever the choice of the second derivative operator. We solved this problem by simply substituting the second derivative of the edge model $\left(E P_{c}\right)$ for the second derivative of the image. Along an edge profile $E P_{c}=\left(s_{c}, A_{c}, \theta_{c}\right)$, the enhanced image is given by:

$$
\widetilde{I}_{0}(u)=I(u)-\frac{1}{s_{c}^{2}} A_{c} C_{s_{c}}^{\prime \prime}(u)
$$

In practice, the deconvolution is separable and it can be applied along the two directions. Depending on its orientation, an edge is enhanced as following:

- $\theta_{c}=\theta_{0}$ or $\theta_{4}: \widetilde{I}_{0}(x)=I(x)-\frac{1}{s_{c}^{2}} A_{c} C_{s_{c}}^{\prime \prime}( \pm x) \quad\left(C_{s_{c}}^{\prime \prime}(+x)\right.$ for $\theta_{0}, C_{s_{c}}^{\prime \prime}(-x)$ for $\left.\theta_{4}\right)$.

- $\theta_{c}=\theta_{2}$ or $\theta_{6}: \widetilde{I}_{0}(y)=I(y)-\frac{1}{s_{c}^{2}} A_{c} C_{s_{c}}^{\prime \prime}( \pm y)$ 
- $\theta_{c}=\theta_{2 k+1_{k \in[0,3]}}($ odd index $): \widetilde{I}_{0}(x, y)=\left\{\begin{array}{l}I(x)-\frac{1}{s_{x}^{2}} A_{c} C_{s_{x}}^{\prime \prime}( \pm x) \\ I(y)-\frac{1}{s_{y}^{2}} A_{c} C_{s_{y}}^{\prime \prime}( \pm y)\end{array}\right.$ with $s_{x}=$ $s_{y}=\frac{1}{\sqrt{2}} s_{c}$

The deconvolution has to be a local process, we choose to limit it so that $95 \%$ of the amplitude of the theoretical profile is corrected. This limit corresponds to a spatial range around the current point of $\left[x_{\min }, x_{\max }\right]$ with $-s_{c} x_{\min }=$ $s_{c} x_{\max }=3, x=0$ being the position of current edge point in the image $I$.

\subsection{Results}

Figure 9 shows an example of enhancement on an image section. The sharpness of the edge profiles is clearly improved. Figures 10 and 11 present a restored real image for which there exists no original image. This example shows that strip borders are not restored. Indeed, strip borders present a configuration of two opposite and close edge profiles. This case of subtractive influence whatever the detection scales does not allow to estimate the profile parameters. Two patterns with two different blurring scales for edge profiles have been designed in figure 13 (original image in figure 12). The enhanced image in figure 14 confirms the ability of the IBEP method to restore different blurs if the equivalent scales are included in the analysis range. Furthermore, this edge enhancement method does not increase the noise which is simply let unchanged.

Blanks of restoration are the results of no edge detection or wrong estimation in the fusion stage. The effects inherent to edge detection by the derivative 
approach, as the rounding of corners (see figure 14), affect the restoration process.

To measure the efficiency of the method, results have been compared with those given by the standard Wiener restoration method. In this method there is always a trade off between restoration and noise or blur. The synthetic image (figure 12) has been locally convolved by a PSF function with two different scale parameters $s_{1}$ et $s_{2}$ to obtain two edge profiles in the image (figure 13). Then gaussian noise of variance $v$ is added. The original image (sharp and clean) is used as reference for quality assessment of the restoration methods. Table 1 presents the results with respect to the degradation parameters for the two methods. Note that only the best result obtained with Wiener restoration has been consigned in the table.

This table shows that in absence of noise, the presented enhancement method gives better results than Wiener filter, this last being not adaptive. As the variance of noise increases, the performances of the enhancement method measured by mean square error decrease. IBEP method does not process in any way the noise and MSE table takes into account the degradation due to the noise as well as the one due to blurring. However, these results prove that the method is stable for images corrupted by noise.

\section{Conclusion}

We have presented a new edge enhancement adaptive method based on the modelization of the edge profiles. Local estimation of the edge model is achieved by a multiscale edge detection process. Edge detection process includes mul- 
tiscale edge detection, multiscale merging and segmentation, this last stage being performed by coincidence assumption and simple thresholding.

This very simple method is efficient especially when the degradation process is not space-invariant and gives better results than Wiener restoration whatever its adjustment. The edge enhancement can be used in cooperation with a denoising method to build a complete restoration system. Our method does not need any parameter adjustment: scales of detection and number of levels have only to be chosen according to scale range in the image to restore.

IBEP has been implemented in an autonomous system composed by a camera and a DSP. The figure 15 shows an example of obtained result. The rate of the system is 0.5 image per second.

The mutual influence of the edges has been taken into account to minimize estimation errors of the model; lack of restoration are mainly due to these errors. Some improvements are expected from another study still in progress in which we try to interpolate the correct values of the neighborhood. An other way for improving could be based for example on ridge detector.

Olivier Laligant received his $\mathrm{PhD}$ degree in 1995 from the Université de Bourgogne, France. He is an assistant professor in the computing, electronic, imaging department (Le2i) at Université de Bourgogne, France. His research interests are focused on multiscale edge detection, merging of data, wavelet transform, and recently motion estimation.

Frédéric Truchetet received the master degree in physics at Dijon University, France, in 1973 and a PhD in electronics at the same university in 1977. He was with Thomson-CSF for two years as a research engineer and he is currently full 
professor and the head of the image processing group at Le2i, Laboratory of Electronic, Computer, and Imaging Sciences, at Université de Bourgogne, France. His research interests are focused on image processing for artificial vision inspection and particularly on wavelet transforms, multiresolution edge detection, and image compression. He is member SPIE, IEEE, and ISIS (a research group of CNRS).

Alain Dupasquier is engineer from CNAM (Conservatoire National des Arts et Métiers) in an industrial group of manufacture of plastic toys.

\section{References}

[1] Y.L. You and M. Kaven. A regularisation approach to joint blur identification and image restoration. IEEE Trans. on Image Processing, vol. 5, no. 3, pp 416-428, Mar. 1996.

[2] M.R. Banham and A.K. Katsaggelos. Digital image restoration. IEEE Signal Processing Magazine, pp. 24-41, Mar. 1997.

[3] Thomas P. Costello and Wasfy B. Mikhael. One-dimensional comparison of wiener filtering and richardon-lucy methods for sectioned restoration of sapce-variant digital images. IEEE on Circuits and Systems - I, pp. 518-522, vol. 49, no 4, April 2002.

[4] D. Kundur and D. Hatzinakos. Blind image deconvolution. IEEE Signal Processing magazine, pp. 43-63, May 1996.

[5] M.R. Banham and A.K. Katsaggelos. Spatially adaptive wavelet-based multiscale image restoration. IEEE Trans. Image Processing, pp. 619633, 5(4), Apr. 1996.

[6] Y.P. Guo, H.P. Lee, and C.L. Teo. Blind restoration of images degraded by space-variant blurs using iterative algorithms for both blur identifi- 
cation and images restoration. Image and Vision Computing, no 5, pp. 399-410, May 1997.

[7] O. Laligant and F. Truchetet. Generalization of shen-castan and cannyderiche filters. SPIE's International Technical Working Group Newsletter, pp. 5,12, vol. 7, n. 2, aug 1998.

[8] J. Shen and S. Castan. An optimal linear operator for edge detection. IEEE Proc. Conf. on Vision and Pattern Recognition, pp. 109-114, 1986.

[9] R. Deriche. Using Canny's criteria to derive a recursively implemented optimal edge detector. International Journal of Computer Vision, vol. 1, no. 2, pp. 167-187, May 1987.

[10] E. Bourennane, P. Gouton, M. Paindavoine, and F. Truchetet. Generalization of canny-deriche filter for detection of noisy exponential edge. Signal Processing, pp. 1317-1328, vol. 82, n. 10, 2002.

[11] J. Canny. A computational approach to edge detection. IEEE Pattern Anal. Mach. Intel., vol. 8, no. 6, pp. 679-698, Nov. 1986.

[12] A. Dupasquier, F. Nicolier, G. Delcroix, F. Truchetet, and O. Laligant. Restoration of blurred images by edge detection. SPIE Intelligent Robots and Computer Vision: Algorithms, Techniques, and Active Vision, pp. 213-222, vol. 3522, Boston, nov. 1998.

[13] A. P. Witkin. Scale-space filtering. Proc. International Joint Conference on A.I., pp. 1019-1022, 1983. 


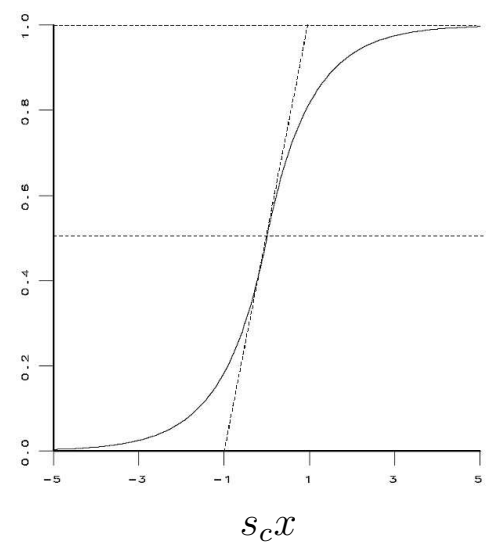

Fig. 1. Edge model defined as an exponential function with $s_{c}>0$

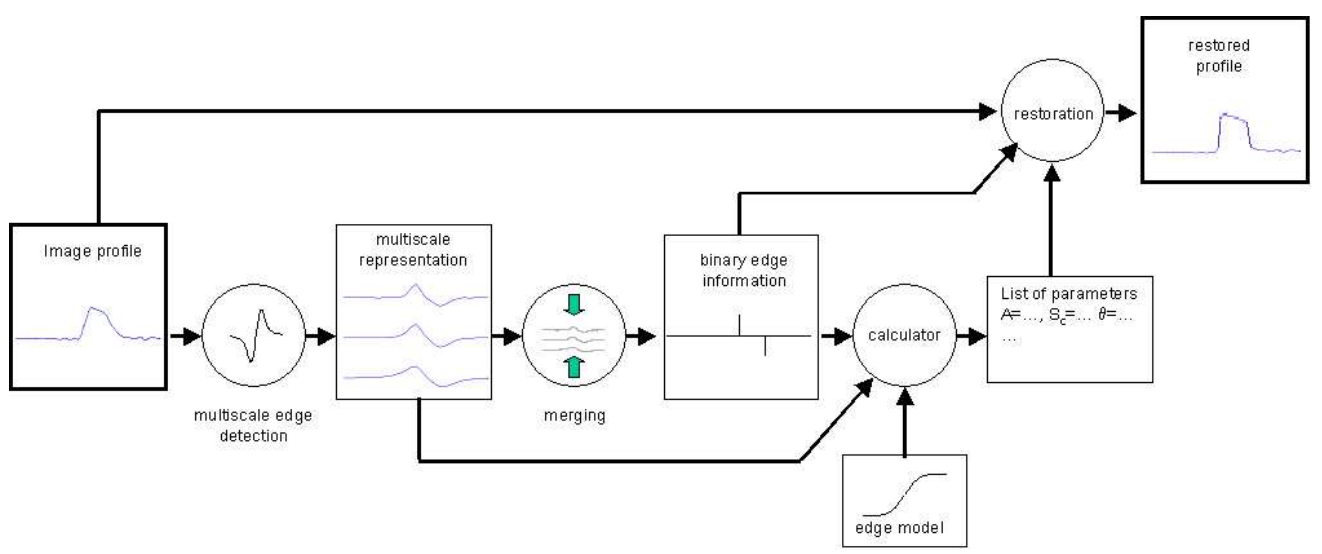

Fig. 2. Overview of the method.

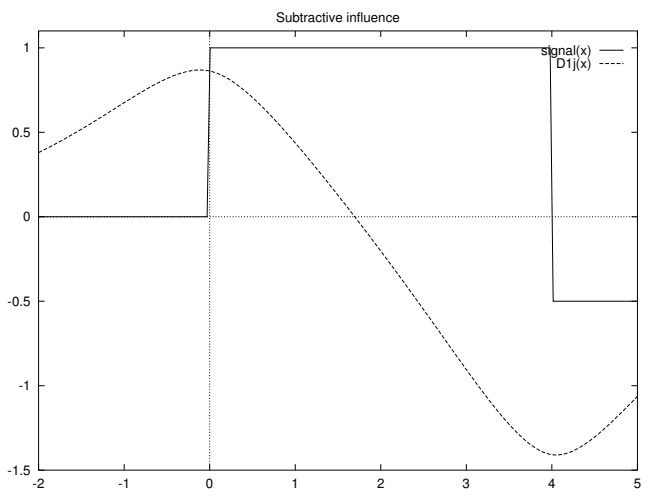

Fig. 3. Subtractive influence of a neighbor edge: the detection amplitude decreases and the highest response maximum moves toward the fine scales as the detector scale decreases. 


\begin{tabular}{|c||ccc|}
\hline \multicolumn{1}{|c||}{} & \multicolumn{3}{|c|}{$s_{1}=0.2, s_{2}=0.4$} \\
\cline { 2 - 4 } \multicolumn{1}{c||}{$M S E$} & $v=0$ & $v=2$ & $v=4$ \\
\hline \hline degraded image & 19.69 & 19.98 & 20.30 \\
\hline Wiener restoration & 9.85 & 11.34 & 11.43 \\
\hline edge enhancement & 8.84 & 11.17 & 12.65 \\
\hline
\end{tabular}

Table 1

Performances of IBEP and Wiener restoration on the "square image". MSE = $\frac{1}{\text { image_size }^{2}} \sum\left[I_{0}(x, y)-\widetilde{I}_{0}(x, y)\right]^{2}$. Degradation parameters: $v$ noise variance, $s_{1}, s_{2}$ blur scales. The best results obtained with Wiener restoration have been consigned. The IBEP method does not take into account the noise and these results show that it is stable against this noise.

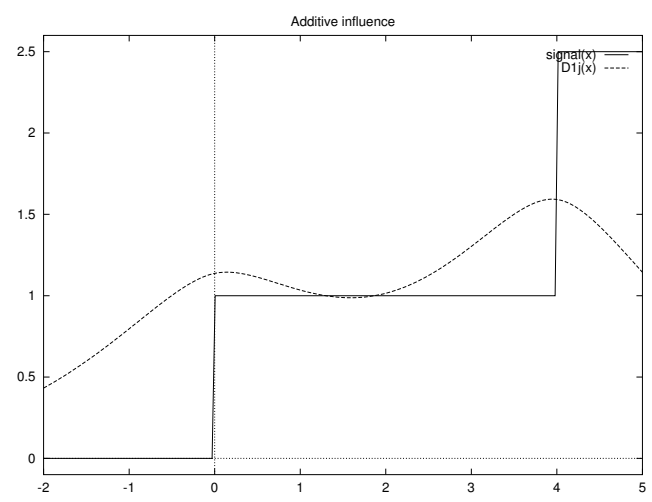

Fig. 4. Additive influence of a neighbor edge: the detection amplitude increases and the highest response maximum moves toward the coarse scales as the detector scale decreases. 
(a)

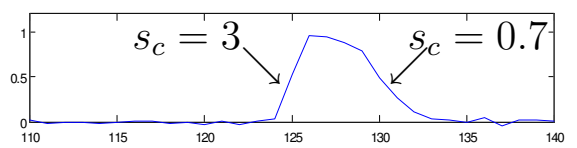

(b)

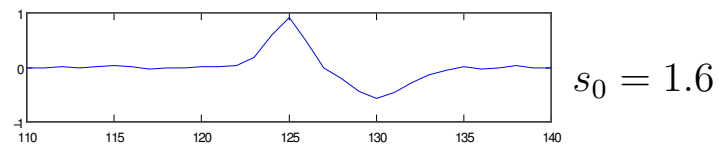

(c)

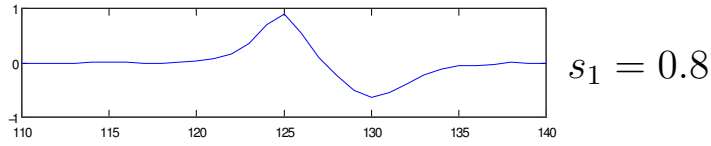

(d)

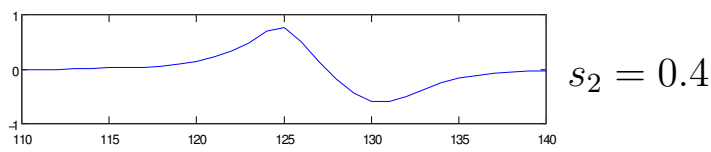

Fig. 5. (a) Edge profiles $\left(s_{c}=3\right.$ and $\left.s_{c}=0.7\right)$ and (b), (c), (d) multiscale analysis $\left(s_{0}=1.6, s_{1}=0.8, s_{2}=0.4\right)$. The best detection is achieved by the first scale for $" s_{c}=3 "$ profile while the second scale performs the best detection for " $s_{c}=0.7$ " profile.

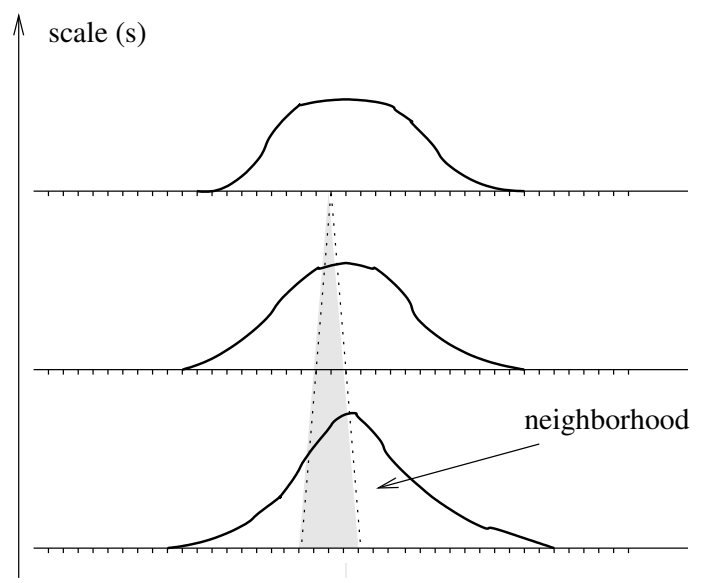

Fig. 6. Fine to coarse approach. 


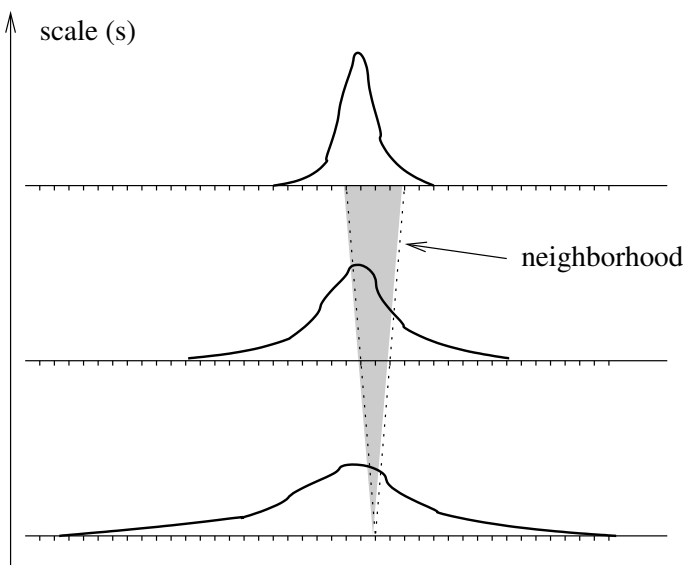

Fig. 7. Coarse to fine approach.

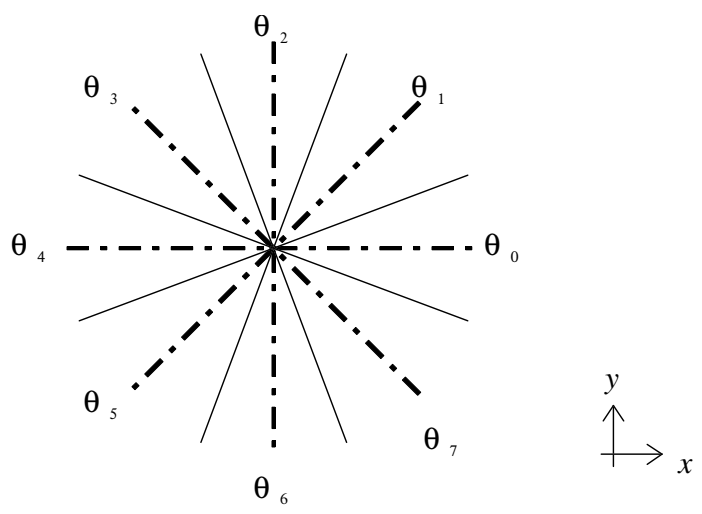

Fig. 8. Gradient orientations according to the Freeman code $\theta_{i}$.

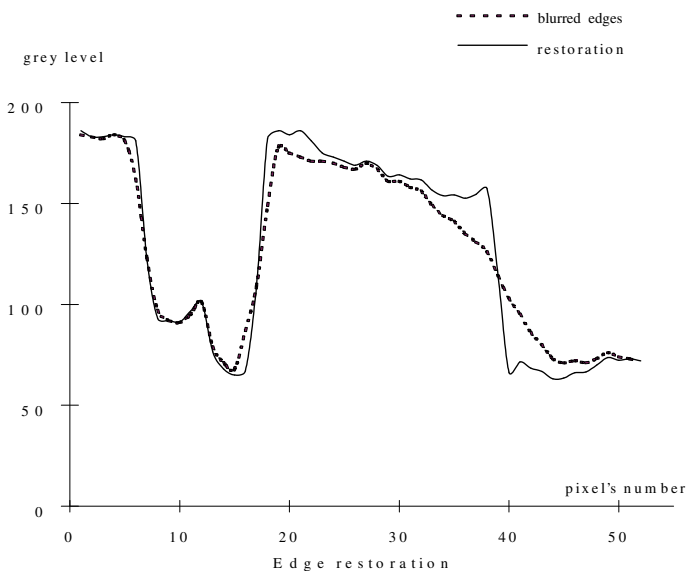

Fig. 9. Image cross section: blur edge profile and its restoration. 


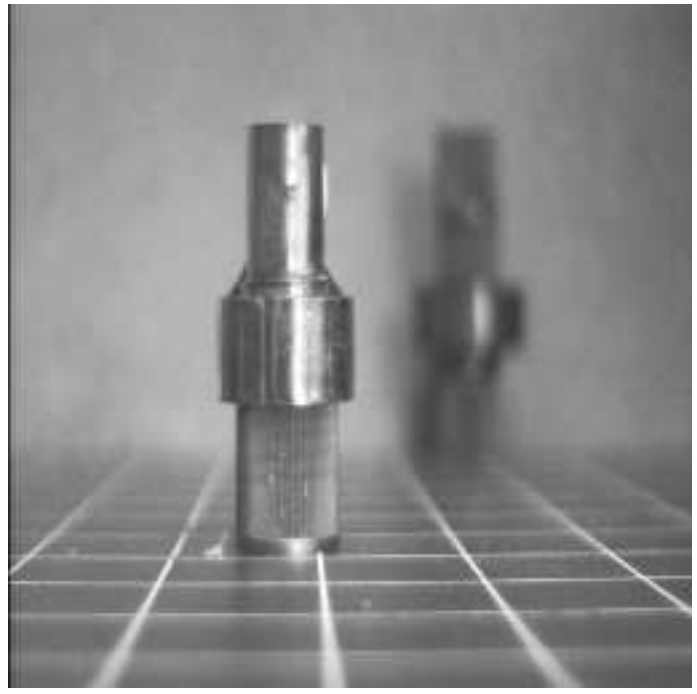

Fig. 10. Original real degraded image.

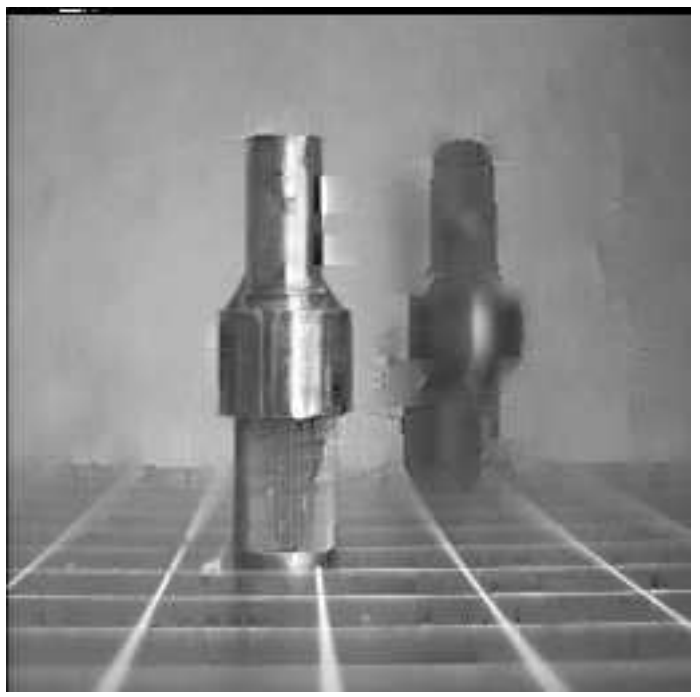

Fig. 11. Restoration of a real degraded image. Lines not restored are under subtractive influence whatever the detection scales. 


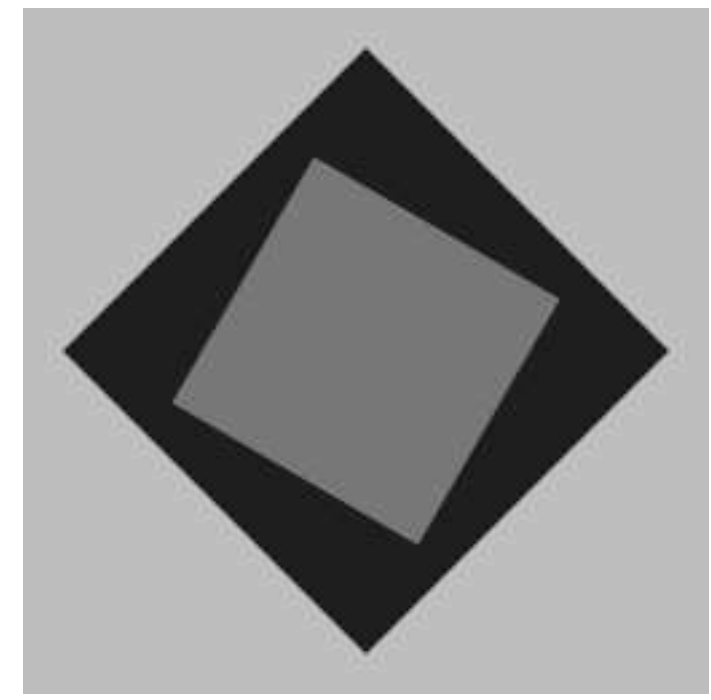

Fig. 12. "Square image": original image.

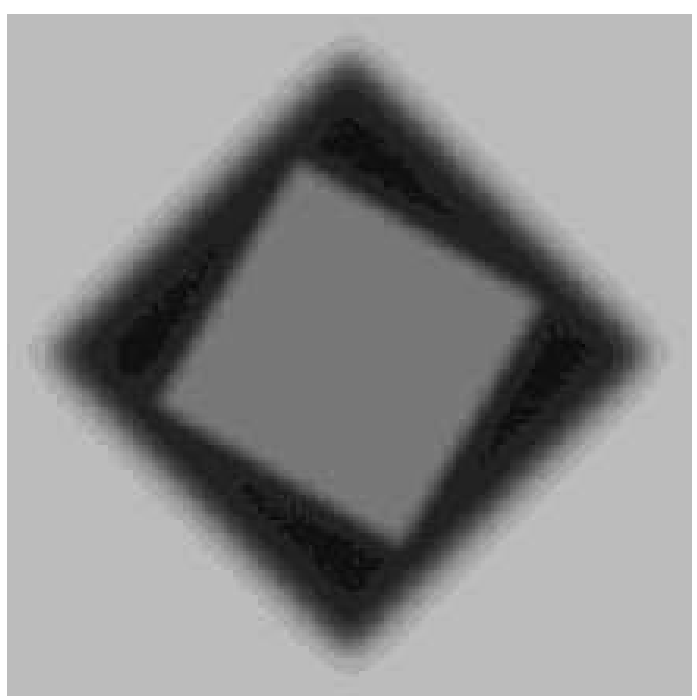

Fig. 13. "Square image": blurred and noisy test synthetic image. 


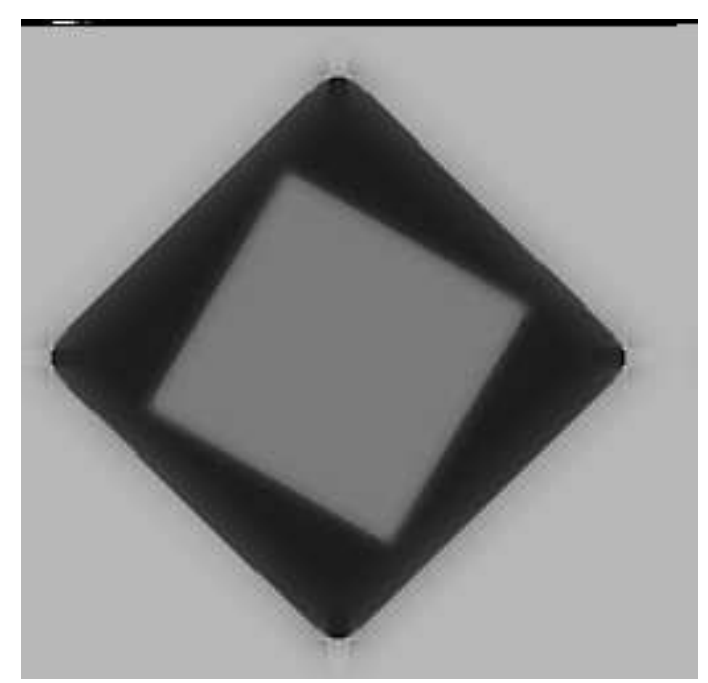

Fig. 14. Restored image by the edge enhancement method.

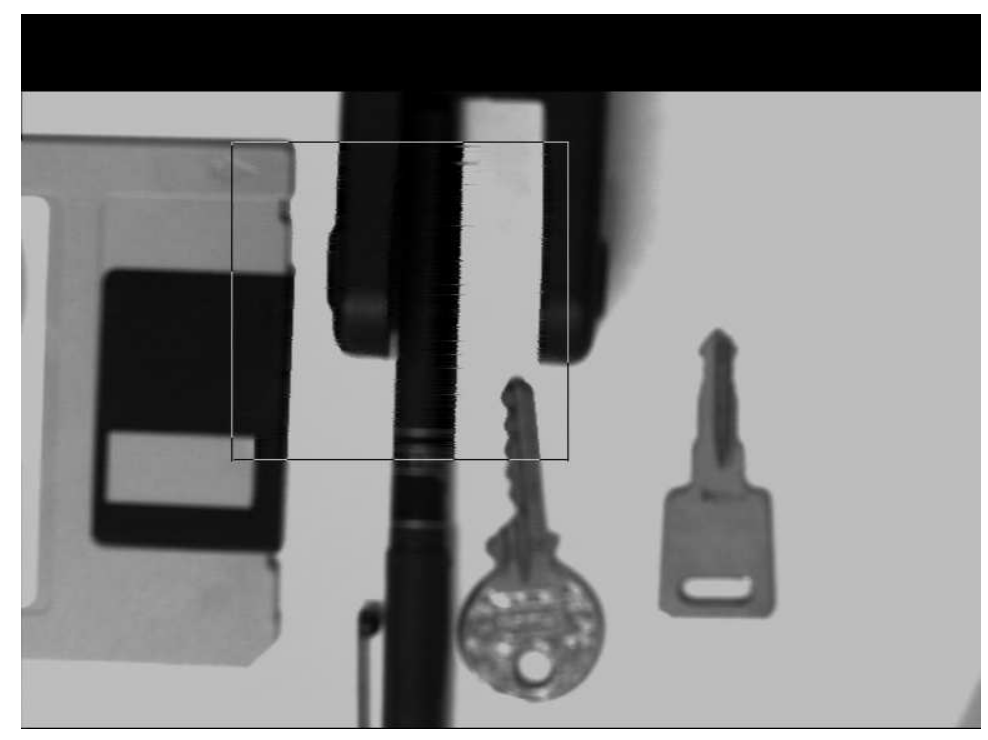

Fig. 15. Restoration by an autonomous system: camera $(740 \times 580$ pixels $)$ and DSP (ADSP $2181-40 \mathrm{MHz})$. This figure shows the blurred image and a piece $(256 \times 256$ pixels) of restoration (inside the square outline). 


\section{A Response of the detectors to the edge model}

The response maximum $y(0)$ of the detectors (eq. 3) to the edge model (eq. 1) is :

$$
\begin{aligned}
y_{n, j}(0) & =f_{n, j} * C_{s_{c}}(0) \\
& =\int_{-\infty}^{+\infty} f_{n, j}(\tau) C_{s_{c}}(-\tau) d \tau \\
& =\int_{-\infty}^{0} f_{n, j}(\tau) d \tau-\int_{-\infty}^{0} f_{n, j}(\tau) \frac{e^{s_{c} \tau}}{2} d \tau+\int_{0}^{\infty} f_{n, j}(\tau) \frac{e^{-s_{c} \tau}}{2} d \tau
\end{aligned}
$$

Symmetry considerations of the filters give:

$$
y_{n, j}(0)=\int_{-\infty}^{0} f_{n, j}(\tau) d \tau-\int_{-\infty}^{0} f_{n, j}(\tau) e^{s_{c} \tau} d \tau
$$

For $x<0$, the filters are defined by:

$$
f_{n, j}(x)=-\operatorname{sign}(x)^{n+1} \frac{s_{j}^{n+1}}{n !} x^{n} e^{s_{j} x}
$$

Hence the second term can be interpreted as the integral of a new filter $f_{n, j+c}$ with a new scale $s_{j}+s_{c}$ and can be written:

$$
y_{n, j}(0)=\int_{-\infty}^{0} f_{n, j}(\tau) d \tau-\frac{s_{j}^{n+1}}{\left(s_{j}+s_{c}\right)^{n+1}} \int_{-\infty}^{0} f_{n, j+c}(\tau) d \tau
$$

Finally, introducing the regularization (integral) operator, we obtain:

$$
\begin{aligned}
y_{n, j}(0) & =h(0)-\frac{s_{j}^{n+1}}{\left(s_{j}+s_{c}\right)^{n+1}} h_{n, j+c}(0) \\
& =1-\frac{s_{j}^{n+1}}{\left(s_{j}+s_{c}\right)^{n+1}}
\end{aligned}
$$




\section{B Localization}

$$
L(f)=\frac{\left|\int_{-\infty}^{+\infty} C_{s_{c}}^{\prime}(-\tau) f^{\prime}(\tau) d \tau\right|}{\left(\int_{-\infty}^{+\infty} f^{\prime 2}(\tau) d \tau\right)^{\frac{1}{2}}}
$$

We write:

$$
f_{n, j}(x)_{x \geqslant 0}=-a_{n} x^{n} e^{-s_{j}|x|}
$$

with

$$
a_{n}=\operatorname{sign}(x)^{n+1} \frac{s_{j}^{n+1}}{n !}=\frac{s_{j}^{n+1}}{n !} \quad \text { for } x \geqslant 0
$$

We have to take into account the 0-order discontinuity (at the abscissa $x=0$ ) for $n=0$. Then for $n=0$ :

$$
\begin{gathered}
\begin{aligned}
\int_{-\infty}^{+\infty} C_{s_{c}}^{\prime}(-\tau) f^{\prime}(\tau) d \tau & =2 \int_{0}^{+\infty}-s_{c} \frac{e^{-s_{c} \tau}}{2} a_{0} s_{j} \tau^{0} e^{-s_{j} \tau} d \tau \\
& =\frac{s_{j}^{2} s_{c}}{s_{j}+s_{c}} \\
\int_{-\infty}^{+\infty} f^{\prime 2}(\tau) d \tau & =2 \int_{0}^{+\infty} s_{j}^{4} e^{-2 s_{j} \tau} d \tau \\
& =-2 s_{j}^{4}\left[\frac{1}{2 s_{j}} e^{-2 s_{j} \tau}\right]_{0}^{+\infty} \\
& =s_{j}^{3}
\end{aligned}
\end{gathered}
$$

And more generally for $n \in\{1,2, \ldots\}$ : 


$$
\begin{gathered}
\int_{-\infty}^{+\infty} C_{s_{c}}^{\prime}(-\tau) f^{\prime}(\tau) d \tau=2 \int_{0}^{+\infty}-s_{c} \frac{e^{-s_{c} \tau}}{2} a_{n}\left(-n \tau^{n-1}+s_{j} \tau^{n}\right) e^{-s_{j} \tau} d \tau \\
=a_{n} s_{c} n \int_{0}^{+\infty} \tau^{n-1} e^{-\left(s_{j}+s_{c}\right) \tau} d \tau-a_{n} s_{c} s_{j} \int_{0}^{+\infty} \tau^{n} e^{-\left(s_{j}+s_{c}\right) \tau} d \tau \\
=a_{n} n ! \frac{s_{c}}{\left(s_{j}+s_{c}\right)^{n}}-a_{n} n ! s_{j} \frac{s_{c}}{\left(s_{j}+s_{c}\right)^{n+1}} \\
=a_{n} n ! \frac{s_{c}^{2}}{\left(s_{j}+s_{c}\right)^{n+1}} \\
\int_{-\infty}^{+\infty} f^{\prime 2}(\tau) d \tau=2 \int_{0}^{+\infty} a_{n}^{2} e^{-2 s_{j} \tau}\left(s_{j} \tau^{n}-n \tau^{n-1}\right)^{2} d \tau \\
=2 a_{n}^{2}\left[\int_{0}^{+\infty} s_{j}^{2} \tau^{2 n} e^{-2 s_{j} \tau} d \tau-\int_{0}^{+\infty} 2 s_{j} n \tau^{2 n-1} e^{-2 s_{j} \tau} d \tau\right. \\
\left.+\int_{0}^{+\infty} n^{2} 2 \tau^{2 n-2} e^{-2 s_{j} \tau} d \tau\right] \\
=2 a_{n}^{2}\left[(2 n) ! \frac{s_{j}^{2}}{\left(2 s_{j}\right)^{2 n+1}}-(2 n-1) ! \frac{2 s_{j} n}{\left(2 s_{j}\right)^{2 n}}+(2 n-2) ! \frac{n^{2}}{\left(2 s_{j}\right)^{2 n-1}}\right] \\
=\frac{a_{n}^{2}}{2\left(2 s_{j}\right)^{2 n-1}}\left[(2 n) !-4 n(2 n-1) !+4 n^{2}(2 n-2) !\right] \\
=\frac{a_{n}^{2}}{\left(2 s_{j}\right)^{2 n-1}} \frac{(2 n) !}{2(2 n-1)}
\end{gathered}
$$

Finally:

$$
\begin{aligned}
L\left(f_{0, j}\right)_{j \in \mathbf{Z}} & =\frac{s_{c}}{s_{j}+s_{c}} \sqrt{s_{j}} \\
L\left(f_{n, j}\right)_{n \in\{1,2, \ldots\}, j \in \mathbf{Z}} & =\frac{s_{c}^{2}}{\left(s_{j}+s_{c}\right)^{n+1}} \sqrt{\frac{2 n-1}{s_{j}(2 n) !}}\left(2 s_{j}\right)^{n} n !
\end{aligned}
$$

Remark 2 Replacing $n$ ! (2n!) by the $\Gamma_{n+1}\left(\Gamma_{2 n+1}\right)$ function extends the relation B.8 to real n.

\section{Signal over noise ratio}

$$
\Sigma(f)=\frac{\left|\int_{-\infty}^{+\infty} C_{s_{c}}(-\tau) f(\tau) d \tau\right|}{\left(\int_{-\infty}^{+\infty} f^{2}(\tau) d \tau\right)^{\frac{1}{2}}}
$$




$$
\begin{aligned}
\int_{-\infty}^{+\infty} f^{2}(\tau) d \tau & =2 a_{n}^{2} \int_{0}^{+\infty} \tau^{2 n} e^{-2 s_{j} \tau} d \tau \\
& =2 a_{n}^{2} \frac{(2 n) !}{\left(2 s_{j}\right)^{2 n+1}} \\
& =2 \frac{s_{j}^{n+1}}{\left(2 s_{j}\right)^{2 n+1}} \frac{(2 n) !}{n !}
\end{aligned}
$$

Combining C.1 and the result A.3 of A.1 we obtain:

$$
\Sigma\left(f_{n, j}\right)_{n \in\{0,1,2, \ldots\}, j \in \mathbf{Z}}=\frac{\left(s_{j}+s_{c}\right)^{n+1}-s_{j}^{n+1}}{\left(s_{j}+s_{c}\right)^{n+1}} \frac{2^{n} n !}{\sqrt{s_{j}(2 n) !}}
$$

Remark 3 Replacing $n$ ! (2n!) by the $\Gamma_{n+1}\left(\Gamma_{2 n+1}\right)$ function extends the relation C.2 to real $n$.

\section{Scale of the detectors for maximum in localization}

For a given edge scale $s_{c}$, we search for the scale $s_{j}$ leading to the maximum in localization. This particular scale is denoted $S_{n}$.

The maximum in localization corresponds to :

$$
\left(\frac{d L\left(f_{n, j}\right)}{d s_{j}}\right)_{s_{j}=S_{n}}=0
$$

Therefore for $n \in\{1,2, \ldots\}$

$$
\begin{aligned}
\frac{d L\left(f_{n, j}\right)}{d s_{j}} & =\frac{d}{d s_{j}}\left(\frac{s_{c}^{2}}{\left(s_{j}+s_{c}\right)^{n+1}} \sqrt{\frac{2 n-1}{s_{j}(2 n) !}}\left(2 s_{j}\right)^{n} n !\right) \\
& =n ! 2^{n} s_{c}^{2} \sqrt{\frac{2 n-1}{(2 n) !}} \frac{d}{d s_{j}}\left(\frac{s_{j}^{n-\frac{1}{2}}}{\left(s_{j}+s_{c}\right)^{n+1}}\right)
\end{aligned}
$$


Simplifying, $\frac{d L\left(f_{n, j}\right)}{d s_{j}}=0$

$$
\begin{aligned}
& \Rightarrow \frac{\left(n-\frac{1}{2}\right) s_{j}^{n-\frac{3}{2}}\left(s_{j}+s_{c}\right)^{n+1}-(n+1)\left(s_{j}+s_{c}\right)^{n} s_{j}^{n-\frac{1}{2}}}{\left(s_{j}+s_{c}\right)^{2 n+2}}=0 \\
& \Rightarrow\left(n-\frac{1}{2}\right) s_{j}^{-1}\left(s_{j}+s_{c}\right)-(n+1)=0
\end{aligned}
$$

Finally

$$
\forall s_{c}>0, n \in\{1,2, \ldots\} \quad \frac{d L\left(f_{n, j}\right)}{d s_{j}}=0 \Rightarrow s_{j}=\frac{1}{3}(2 n-1) s_{c}
$$

This scale is denoted $S_{n}$. The demonstration of $S_{0}$ is trivial. 